\title{
Morrey spaces related to certain nonnegative potentials and fractional integrals on the Heisenberg groups
}

Hua Wang ${ }^{1,2^{*}}$ (D)

\begin{tabular}{l}
\hline${ }^{\text {"Correspondence: }}$ \\
wanghua@pku.edu.cn \\
'School of Mathematics and \\
Systems Science, Xinjiang \\
University, Urumqi, P.R. China \\
${ }^{2}$ Department of Mathematics and \\
Statistics, Memorial University, St. \\
John's, Canada
\end{tabular}

\begin{abstract}
Let $\mathcal{L}=-\Delta_{\mathbb{H}^{n}}+V$ be a Schrödinger operator on the Heisenberg group $\mathbb{H}^{n}$, where $\Delta_{\mathbb{H}^{n}}$ is the sub-Laplacian on $\mathbb{H}^{n}$ and the nonnegative potential $V$ belongs to the reverse Hölder class $R H_{s}$ with $s \geq Q / 2$. Here $Q=2 n+2$ is the homogeneous dimension of $\mathbb{H}^{n}$. For given $\alpha \in(0, Q)$, the fractional integrals associated to the Schrödinger operator $\mathcal{L}$ is defined by $\mathcal{I}_{\alpha}=\mathcal{L}^{-\alpha / 2}$. In this article, we first introduce the Morrey space $L_{\rho, \infty}^{p, \kappa}\left(\mathbb{H}^{n}\right)$ and weak Morrey space $W L_{\rho, \infty}^{p, \kappa}\left(\mathbb{H}^{n}\right)$ related to the nonnegative potential $V$. Then we establish the boundedness of fractional integrals $\mathcal{L}^{-\alpha / 2}$ on these new spaces. Furthermore, in order to deal with certain extreme cases, we also introduce the spaces $\mathrm{BMO}_{\rho, \infty}\left(\mathbb{H}^{n}\right)$ and $\mathcal{C}_{\rho, \infty}^{\beta}\left(\mathbb{H}^{n}\right)$ with exponent $\beta \in(0,1]$.
\end{abstract}

MSC: Primary 42B20; 35J10; secondary 22E25; 22E30

Keywords: Schrödinger operator; Fractional integrals; Heisenberg group; Morrey spaces; Reverse Hölder class

\section{Introduction}

\subsection{Heisenberg group $\mathbb{H}^{n}$}

The Heisenberg group $\mathbb{H}^{n}$ is a nilpotent Lie group with underlying manifold $\mathbb{C}^{n} \times \mathbb{R}$. The group structure (the multiplication law) is given by

$$
(z, t) \cdot\left(z^{\prime}, t^{\prime}\right):=\left(z+z^{\prime}, t+t^{\prime}+2 \operatorname{Im}\left(z \cdot \overline{z^{\prime}}\right)\right),
$$

where $z=\left(z_{1}, z_{2}, \ldots, z_{n}\right), z^{\prime}=\left(z_{1}^{\prime}, z_{2}^{\prime}, \ldots, z_{n}^{\prime}\right) \in \mathbb{C}^{n}$, and

$$
z \cdot \overline{z^{\prime}}:=\sum_{j=1}^{n} z_{j} \overline{z_{j}^{\prime}}
$$

It can be easily seen that the inverse element of $u=(z, t)$ is $u^{-1}=(-z,-t)$, and the identity is the origin $(0,0)$. The Lie algebra of left-invariant vector fields on $\mathbb{H}^{n}$ is spanned by

$$
\begin{cases}X_{j}=\frac{\partial}{\partial x_{j}}+2 y_{j} \frac{\partial}{\partial t}, & j=1,2, \ldots, n, \\ Y_{j}=\frac{\partial}{\partial y_{j}}-2 x_{j} \frac{\partial}{\partial t}, & j=1,2, \ldots, n, \\ T=\frac{\partial}{\partial t} .\end{cases}
$$

(c) The Author(s) 2019. This article is distributed under the terms of the Creative Commons Attribution 4.0 International License (http://creativecommons.org/licenses/by/4.0/), which permits unrestricted use, distribution, and reproduction in any medium, provided you give appropriate credit to the original author(s) and the source, provide a link to the Creative Commons license, and indicate if changes were made. 
All non-trivial commutation relations are given by

$$
\left[X_{j}, Y_{j}\right]=-4 T, \quad j=1,2, \ldots, n
$$

The sub-Laplacian $\Delta_{\mathbb{H}^{n}}$ is defined by

$$
\Delta_{\mathbb{H}^{n}}:=\sum_{j=1}^{n}\left(X_{j}^{2}+Y_{j}^{2}\right)
$$

The dilations on $\mathbb{H}^{n}$ have the following form:

$$
\delta_{a}(z, t):=\left(a z, a^{2} t\right), \quad a>0
$$

For given $(z, t) \in \mathbb{H}^{n}$, the homogeneous norm of $(z, t)$ is given by

$$
|(z, t)|:=\left(|z|^{4}+t^{2}\right)^{1 / 4}
$$

Observe that $\left|(z, t)^{-1}\right|=|(z, t)|$ and

$$
\left|\delta_{a}(z, t)\right|=\left(|a z|^{4}+\left(a^{2} t\right)^{2}\right)^{1 / 4}=a|(z, t)|
$$

In addition, this norm $|\cdot|$ satisfies the triangle inequality and leads to a left-invariant distance $d(u, v)=\left|u^{-1} \cdot v\right|$ for $u=(z, t), v=\left(z^{\prime}, t^{\prime}\right) \in \mathbb{H}^{n}$. The ball of radius $r$ centered at $u$ is denoted by

$$
B(u, r):=\left\{v \in \mathbb{H}^{n}: d(u, v)<r\right\}
$$

The Haar measure on $\mathbb{H}^{n}$ coincides with the Lebesgue measure on $\mathbb{R}^{2 n} \times \mathbb{R}$. The measure of any measurable set $E \subset \mathbb{H}^{n}$ is denoted by $|E|$. For $(u, r) \in \mathbb{H}^{n} \times(0, \infty)$, it can be shown that the volume of $B(u, r)$ is

$$
|B(u, r)|=r^{Q} \cdot|B(0,1)|
$$

where $Q:=2 n+2$ is the homogeneous dimension of $\mathbb{H}^{n}$ and $|B(0,1)|$ is the volume of the unit ball in $\mathbb{H}^{n}$. A direct calculation shows that

$$
|B(0,1)|=\frac{2 \pi^{n+\frac{1}{2}} \Gamma\left(\frac{n}{2}\right)}{(n+1) \Gamma(n) \Gamma\left(\frac{n+1}{2}\right)} .
$$

Given a ball $B=B(u, r)$ in $\mathbb{H}^{n}$ and $\lambda>0$, we shall use the notation $\lambda B$ to denote $B(u, \lambda r)$. Clearly, we have

$$
|B(u, \lambda r)|=\lambda^{Q} \cdot|B(u, r)|
$$

For more information about the harmonic analysis on the Heisenberg groups, we refer the reader to [12, Chapter XII] and [13]. 
Let $V: \mathbb{H}^{n} \rightarrow \mathbb{R}$ be a nonnegative locally integrable function that belongs to the reverse Hölder class $R H_{s}$ for some exponent $1<s<\infty$; i.e., there exists a positive constant $C>0$ such that the reverse Hölder inequality

$$
\left(\frac{1}{|B|} \int_{B} V(w)^{s} d w\right)^{1 / s} \leq C\left(\frac{1}{|B|} \int_{B} V(w) d w\right)
$$

holds for every ball $B$ in $\mathbb{H}^{n}$. For given $V \in R H_{s}$ with $s \geq Q / 2$, we introduce the critical radius function $\rho(u)=\rho(u ; V)$ which is given by

$$
\rho(u):=\sup \left\{r>0: \frac{1}{r^{Q-2}} \int_{B(u, r)} V(w) d w \leq 1\right\}, \quad u \in \mathbb{H}^{n},
$$

where $B(u, r)$ denotes the ball in $\mathbb{H}^{n}$ centered at $u$ and with radius $r$. It is well known that this auxiliary function satisfies $0<\rho(u)<\infty$ for any $u \in \mathbb{H}^{n}$ under the above assumption on $V$ (see [9]). We need the following well-known result concerning the critical radius function (1.2).

Lemma 1.1 ([9]) If $V \in R H_{s}$ with $s \geq Q / 2$, then there exist constants $C_{0} \geq 1$ and $N_{0}>0$ such that, for all $u$ and $v$ in $\mathbb{H}^{n}$,

$$
\frac{1}{C_{0}}\left(1+\frac{\left|v^{-1} u\right|}{\rho(u)}\right)^{-N_{0}} \leq \frac{\rho(v)}{\rho(u)} \leq C_{0}\left(1+\frac{\left|v^{-1} u\right|}{\rho(u)}\right)^{\frac{N_{0}}{N_{0}+1}} .
$$

Lemma 1.1 is due to $\mathrm{Lu}$ [9]. In the setting of $\mathbb{R}^{n}$, this result was given by Shen in [10]. As a straightforward consequence of (1.3), we can see that, for each integer $k \geq 1$, the estimate

$$
1+\frac{2^{k} r}{\rho(v)} \geq \frac{1}{C_{0}}\left(1+\frac{r}{\rho(u)}\right)^{-\frac{N_{0}}{N_{0}+1}}\left(1+\frac{2^{k} r}{\rho(u)}\right)
$$

holds for any $v \in B(u, r)$ with $u \in \mathbb{H}^{n}$ and $r>0, C_{0}$ is the same as in (1.3).

\subsection{Fractional integrals}

First we recall the fractional power of the Laplacian operator on $\mathbb{R}^{n}$. For given $\alpha \in(0, n)$, the classical fractional integral operator $I_{\alpha}^{\Delta}$ (also referred to as the Riesz potential) is defined by

$$
I_{\alpha}^{\Delta}(f):=(-\Delta)^{-\alpha / 2}(f),
$$

where $\Delta$ is the Laplacian operator on $\mathbb{R}^{n}$. If $f \in \mathcal{S}\left(\mathbb{R}^{n}\right)$, then, by virtue of the Fourier transform, we have

$$
\widehat{I_{\alpha}^{\Delta} f}(\xi)=(2 \pi|\xi|)^{-\alpha} \widehat{f}(\xi), \quad \forall \xi \in \mathbb{R}^{n} .
$$

Comparing this to the Fourier transform of $|x|^{-\alpha}, 0<\alpha<n$, we are led to redefine the fractional integral operator $I_{\alpha}^{\Delta}$ by

$$
I_{\alpha}^{\Delta} f(x):=\frac{1}{\gamma(\alpha)} \int_{\mathbb{R}^{n}} \frac{f(y)}{|x-y|^{n-\alpha}} d y,
$$


where

$$
\gamma(\alpha)=\frac{\pi^{\frac{n}{2}} 2^{\alpha} \Gamma\left(\frac{\alpha}{2}\right)}{\Gamma\left(\frac{n-\alpha}{2}\right)}
$$

with $\Gamma(\cdot)$ being the usual gamma function. It is well known that the Hardy-LittlewoodSobolev theorem states that the fractional integral operator $I_{\alpha}^{\Delta}$ is bounded from $L^{p}\left(\mathbb{R}^{n}\right)$ to $L^{q}\left(\mathbb{R}^{n}\right)$ for $0<\alpha<n, 1<p<n / \alpha$ and $1 / q=1 / p-\alpha / n$. Also we know that $I_{\alpha}^{\Delta}$ is bounded from $L^{1}\left(\mathbb{R}^{n}\right)$ to $W L^{q}\left(\mathbb{R}^{n}\right)$ for $0<\alpha<n$ and $q=n /(n-\alpha)$ (see [11]).

Next we are going to discuss the fractional integrals on the Heisenberg group. For given $\alpha \in(0, Q)$ with $Q=2 n+2$, the fractional integral operator $I_{\alpha}$ (also referred to as the Riesz potential) is defined by (see [14])

$$
I_{\alpha}(f):=\left(-\Delta_{\mathbb{H}^{n}}\right)^{-\alpha / 2}(f),
$$

where $\Delta_{\mathbb{H}^{n}}$ is the sub-Laplacian on $\mathbb{H}^{n}$ defined above. Let $f$ and $g$ be integrable functions defined on $\mathbb{H}^{n}$. Define the convolution $f * g$ by

$$
(f * g)(u):=\int_{\mathbb{H}^{h}} f(v) g\left(v^{-1} u\right) d v .
$$

We denote by $H_{s}(u)$ the convolution kernel of heat semigroup $\left\{T_{s}=e^{s \Delta_{\mathbb{H}^{n}}}: s>0\right\}$. Namely,

$$
e^{s \Delta_{\mathbb{H}^{n}}} f(u)=\int_{\mathbb{H}^{n}} H_{s}\left(v^{-1} u\right) f(v) d v .
$$

For any $u=(z, t) \in \mathbb{H}^{n}$, it was proved in [14, Theorem 4.2] that $I_{\alpha}$ can be expressed by the following formula:

$$
\begin{aligned}
I_{\alpha} f(u) & =\frac{1}{\Gamma(\alpha / 2)} \int_{0}^{\infty} e^{s \Delta_{\mathbb{H}} n} f(u) s^{\alpha / 2-1} d s \\
& =\frac{1}{\Gamma(\alpha / 2)} \int_{0}^{\infty}\left(H_{s} * f\right)(u) s^{\alpha / 2-1} d s .
\end{aligned}
$$

Let $V \in R H_{s}$ for $s \geq Q / 2$. For such a potential $V$, we consider the time independent Schrödinger operator on $\mathbb{H}^{n}$ (see [8]),

$$
\mathcal{L}:=-\Delta_{\mathbb{H}^{n}}+V,
$$

and its associated semigroup

$$
\mathcal{T}_{s}^{\mathcal{L}} f(u):=e^{-s \mathcal{L}} f(u)=\int_{\mathbb{H}^{n}} P_{s}(u, v) f(v) d v, \quad f \in L^{2}\left(\mathbb{H}^{n}\right), s>0,
$$

where $P_{s}(u, v)$ denotes the kernel of the operator $e^{-s \mathcal{L}}, s>0$. For any $u=(z, t) \in \mathbb{H}^{n}$, it is well known that the heat kernel $H_{s}(u)$ has the explicit expression

$$
H_{s}(z, t)=(2 \pi)^{-1}(4 \pi)^{-n} \int_{\mathbb{R}}\left(\frac{|\lambda|}{\sinh |\lambda| s}\right)^{n} \exp \left\{-\frac{|\lambda||z|^{2}}{4} \operatorname{coth}|\lambda| s-i \lambda t\right\} d \lambda,
$$


and hence it satisfies the following estimate (see [5] for instance):

$$
0 \leq H_{s}(u) \leq C \cdot s^{-Q / 2} \exp \left(-\frac{|u|^{2}}{A s}\right)
$$

where the constants $C, A>0$ are independent of $s$ and $u \in \mathbb{H}^{n}$. Since $V \geq 0$, by the Trotter product formula and (1.8), one has

$$
0 \leq P_{s}(u, v) \leq H_{s}\left(v^{-1} u\right) \leq C \cdot s^{-Q / 2} \exp \left(-\frac{\left|v^{-1} u\right|^{2}}{A s}\right), \quad s>0 .
$$

Moreover, this estimate (1.9) can be improved when $V$ belongs to the reverse Hölder class $R H_{s}$ for some $s \geq Q / 2$. The auxiliary function $\rho(u)$ arises naturally in this context.

Lemma 1.2 Let $V \in R H_{s}$ with $s \geq Q / 2$, and let $\rho(u)$ be the auxiliary function determined by $V$. For every positive integer $N \geq 1$, there exists a positive constant $C_{N}>0$ such that, for all $u$ and $v$ in $\mathbb{H}^{n}$,

$$
0 \leq P_{s}(u, v) \leq C_{N} \cdot s^{-Q / 2} \exp \left(-\frac{\left|v^{-1} u\right|^{2}}{A s}\right)\left(1+\frac{\sqrt{s}}{\rho(u)}+\frac{\sqrt{s}}{\rho(v)}\right)^{-N}, \quad s>0 .
$$

This estimate of $P_{s}(u, v)$ is better than (1.9), which was given by Lin and Liu in [8, Lemma 7].

Inspired by (1.6) and (1.7), for given $\alpha \in(0, Q)$, the $\mathcal{L}$-fractional integral operator or $\mathcal{L}$ Riesz potential on the Heisenberg group is defined by (see [6] and [7])

$$
\begin{aligned}
\mathcal{I}_{\alpha}(f)(u) & :=\mathcal{L}^{-\alpha / 2} f(u) \\
& =\frac{1}{\Gamma(\alpha / 2)} \int_{0}^{\infty} e^{-s \mathcal{L}} f(u) s^{\alpha / 2-1} d s .
\end{aligned}
$$

Recall that in the setting of $\mathbb{R}^{n}$, this integral operator was first introduced by Dziubański et al. [3]. In this article we shall be interested in the behavior of the fractional integral operator $\mathcal{I}_{\alpha}$ associated to Schrödinger operator on $\mathbb{H}^{n}$. For $1 \leq p<\infty$, the Lebesgue space $L^{p}\left(\mathbb{H}^{n}\right)$ is defined to be the set of all measurable functions $f$ on $\mathbb{H}^{n}$ such that

$$
\|f\|_{L^{p}\left(\mathbb{H}^{n}\right)}:=\left(\int_{\mathbb{H}^{n}}|f(u)|^{p} d u\right)^{1 / p}<\infty
$$

The weak Lebesgue space $W L^{p}\left(\mathbb{H}^{n}\right)$ consists of all measurable functions $f$ on $\mathbb{H}^{n}$ such that

$$
\|f\|_{W L^{p}\left(\mathbb{H}^{n}\right)}:=\sup _{\lambda>0} \lambda \cdot\left|\left\{u \in \mathbb{H}^{n}:|f(u)|>\lambda\right\}\right|^{1 / p}<\infty .
$$

Now we are going to establish strong-type and weak-type estimates of the $\mathcal{L}$-fractional integral operator $\mathcal{I}_{\alpha}$ on the Lebesgue spaces. We first claim that the estimate

$$
\left|\mathcal{I}_{\alpha} f(u)\right| \leq C \int_{\mathbb{H}^{n}}|f(v)| \frac{1}{\left|v^{-1} u\right|^{Q-\alpha}} d v=C\left(|f| *|\cdot|^{\alpha-Q}\right)(u)
$$


holds for all $u \in \mathbb{H}^{n}$. Let us verify (1.10). To do so, denote by $\mathcal{K}_{\alpha}(u, v)$ the kernel of the fractional integral operator $\mathcal{I}_{\alpha}$. Then we have

$$
\begin{aligned}
\int_{\mathbb{H}^{n}} \mathcal{K}_{\alpha}(u, v) f(v) d v & =\mathcal{I}_{\alpha} f(u)=\mathcal{L}^{-\alpha / 2} f(u) \\
& =\frac{1}{\Gamma(\alpha / 2)} \int_{0}^{\infty} e^{-s \mathcal{L}} f(u) s^{\alpha / 2-1} d s \\
& =\int_{0}^{\infty}\left[\frac{1}{\Gamma(\alpha / 2)} \int_{\mathbb{H}^{n}} P_{s}(u, v) f(v) d v\right] s^{\alpha / 2-1} d s \\
& =\int_{\mathbb{H}^{n}}\left[\frac{1}{\Gamma(\alpha / 2)} \int_{0}^{\infty} P_{s}(u, v) s^{\alpha / 2-1} d s\right] f(v) d v
\end{aligned}
$$

Hence,

$$
\mathcal{K}_{\alpha}(u, v)=\frac{1}{\Gamma(\alpha / 2)} \int_{0}^{\infty} P_{s}(u, v) s^{\alpha / 2-1} d s .
$$

Moreover, by using (1.9), we can deduce that

$$
\begin{aligned}
\left|\mathcal{K}_{\alpha}(u, v)\right| & \leq \frac{C}{\Gamma(\alpha / 2)} \int_{0}^{\infty} \exp \left(-\frac{\left|v^{-1} u\right|^{2}}{A s}\right) s^{\alpha / 2-Q / 2-1} d s \\
& \leq \frac{C}{\Gamma(\alpha / 2)} \cdot \frac{1}{\left|v^{-1} u\right|^{Q-\alpha}} \int_{0}^{\infty} e^{-t} t^{(Q / 2-\alpha / 2)-1} d t \\
& =C \cdot \frac{\Gamma(Q / 2-\alpha / 2)}{\Gamma(\alpha / 2)} \cdot \frac{1}{\left|v^{-1} u\right|^{Q-\alpha}}
\end{aligned}
$$

where in the second step we have used a change of variables. Thus (1.10) holds. According to Theorems 4.4 and 4.5 in [14], we get the Hardy-Littlewood-Sobolev theorem on the Heisenberg group.

Theorem 1.3 Let $0<\alpha<Q$ and $1 \leq p<Q / \alpha$. Define $1<q<\infty$ by the relation $1 / q=1 / p-$ $\alpha / Q$. Then the following statements are valid:

(1) if $p>1$, then $\mathcal{I}_{\alpha}$ is bounded from $L^{p}\left(\mathbb{H}^{n}\right)$ to $L^{q}\left(\mathbb{H}^{n}\right)$;

(2) if $p=1$, then $\mathcal{I}_{\alpha}$ is bounded from $L^{1}\left(\mathbb{H}^{n}\right)$ to $W L^{q}\left(\mathbb{H}^{n}\right)$.

The organization of this paper is as follows. In Sect. 2, we will give the definitions of Morrey space and weak Morrey space and state our main results: Theorems 2.3, 2.4, and 2.5. Section 3 is devoted to proving the boundedness of the fractional integral operator in the context of Morrey spaces. We will study certain extreme cases in Sect. 4. Throughout this paper, $C$ represents a positive constant that is independent of the main parameters, but may be different from line to line, and a subscript is added when we wish to make clear its dependence on the parameter in the subscript. We also use $a \approx b$ to denote the equivalence of $a$ and $b$; that is, there exist two positive constants $C_{1}, C_{2}$ independent of $a$, $b$ such that $C_{1} a \leq b \leq C_{2} a$.

\section{Main results}

In this section, we introduce some types of Morrey spaces related to the nonnegative potential $V$ on $\mathbb{H}^{n}$, and then give our main results. 
Definition 2.1 Let $\rho$ be the auxiliary function determined by $V \in R H_{s}$ with $s \geq Q / 2$. Let $1 \leq p<\infty$ and $0 \leq \kappa<1$. For given $0<\theta<\infty$, the Morrey space $L_{\rho, \theta}^{p, \kappa}\left(\mathbb{H}^{n}\right)$ is defined to be the set of all $p$-locally integrable functions $f$ on $\mathbb{H}^{n}$ such that

$$
\left(\frac{1}{|B|^{\kappa}} \int_{B}|f(u)|^{p} d u\right)^{1 / p} \leq C \cdot\left(1+\frac{r}{\rho\left(u_{0}\right)}\right)^{\theta}
$$

for every ball $B=B\left(u_{0}, r\right)$ in $\mathbb{H}^{n}$. A norm for $f \in L_{\rho, \theta}^{p, \kappa}\left(\mathbb{H}^{n}\right)$, denoted by $\|f\|_{L_{\rho, \theta}^{p, \kappa}\left(\mathbb{H}^{n}\right)}$, is given by the infimum of the constants in (2.1), or equivalently,

$$
\|f\|_{L_{\rho, \theta}^{p, \kappa}\left(\mathbb{H}^{n}\right)}:=\sup _{B\left(u_{0}, r\right)}\left(1+\frac{r}{\rho\left(u_{0}\right)}\right)^{-\theta}\left(\frac{1}{|B|^{\kappa}} \int_{B}|f(u)|^{p} d u\right)^{1 / p}<\infty,
$$

where the supremum is taken over all balls $B=B\left(u_{0}, r\right)$ in $\mathbb{H}^{n}, u_{0}$ and $r$ denote the center and radius of $B$, respectively. Define

$$
L_{\rho, \infty}^{p, \kappa}\left(\mathbb{H}^{n}\right):=\bigcup_{\theta>0} L_{\rho, \theta}^{p, \kappa}\left(\mathbb{H}^{n}\right) .
$$

For any given $f \in L_{\rho, \infty}^{p, \kappa}\left(\mathbb{H}^{n}\right)$, let

$$
\theta^{*}:=\inf \left\{\theta>0: f \in L_{\rho, \theta}^{p, \kappa}\left(\mathbb{H}^{n}\right)\right\} .
$$

Now define

$$
\|f\|_{\star}=\|f\|_{L_{\rho, \infty}^{p, \kappa}\left(\mathbb{H}^{n}\right)}:=\|f\|_{L_{\rho, \theta^{*}}^{p, \kappa}\left(\mathbb{H}^{n}\right)} .
$$

It is easy to check that $\|\cdot\|_{\star}$ satisfies the axioms of a norm; i.e., that for $f, g \in L_{\rho, \infty}^{p, \kappa}\left(\mathbb{H}^{n}\right)$ and $\lambda \in \mathbb{R}$ we have

- $\|f\|_{\star} \geq 0$

- $\|f\|_{\star}=0 \Leftrightarrow f=0$

- $\|\lambda f\|_{\star}=|\lambda|\|f\|_{\star} ;$

- $\|f+g\|_{\star} \leq\|f\|_{\star}+\|g\|_{\star}$.

Definition 2.2 Let $\rho$ be the auxiliary function determined by $V \in R H_{s}$ with $s \geq Q / 2$. Let $1 \leq p<\infty$ and $0 \leq \kappa<1$. For given $0<\theta<\infty$, the weak Morrey space $W L_{\rho, \theta}^{p, \kappa}\left(\mathbb{H}^{n}\right)$ is defined to be the set of all measurable functions $f$ on $\mathbb{H}^{n}$ such that

$$
\frac{1}{|B|^{\kappa / p}} \sup _{\lambda>0} \lambda \cdot|\{u \in B:|f(u)|>\lambda\}|^{1 / p} \leq C \cdot\left(1+\frac{r}{\rho\left(u_{0}\right)}\right)^{\theta}
$$

for every ball $B=B\left(u_{0}, r\right)$ in $\mathbb{H}^{n}$, or equivalently,

$$
\|f\|_{W L_{\rho, \theta}^{p, \kappa}\left(\mathbb{H}^{n}\right)}:=\sup _{B\left(u_{0}, r\right)}\left(1+\frac{r}{\rho\left(u_{0}\right)}\right)^{-\theta} \frac{1}{|B|^{\kappa / p}} \sup _{\lambda>0} \lambda \cdot|\{u \in B:|f(u)|>\lambda\}|^{1 / p}<\infty .
$$

Correspondingly, we define

$$
W L_{\rho, \infty}^{p, \kappa}\left(\mathbb{H}^{n}\right):=\bigcup_{\theta>0} W L_{\rho, \theta}^{p, \kappa}\left(\mathbb{H}^{n}\right) .
$$


For any given $f \in W L_{\rho, \infty}^{p, \kappa}\left(\mathbb{H}^{n}\right)$, let

$$
\theta^{* *}:=\inf \left\{\theta>0: f \in W L_{\rho, \theta}^{p, \kappa}\left(\mathbb{H}^{n}\right)\right\}
$$

Similarly, we define

$$
\|f\|_{\star \star}=\|f\|_{W L_{\rho, \infty}^{p, \kappa}\left(\mathbb{H}^{n}\right)}:=\|f\|_{W L_{\rho, \theta^{* *}}^{p, \kappa}\left(\mathbb{H}^{n}\right)^{.}} .
$$

By definition, we can easily show that $\|\cdot\|_{\star \star}$ satisfies the axioms of a (quasi)norm, and $W L_{\rho, \infty}^{p, \kappa}\left(\mathbb{H}^{n}\right)$ is a (quasi)normed linear space. Obviously, if we take $\theta=0$ or $V \equiv 0$, then this Morrey space (or weak Morrey space) is just the Morrey space $L^{p, \kappa}\left(\mathbb{H}^{n}\right)$ (or $W L^{p, \kappa}\left(\mathbb{H}^{n}\right)$ ), which was defined by Guliyev et al. [4]. Moreover, according to the above definitions, one has

$$
\left\{\begin{array}{l}
L^{p, \kappa}\left(\mathbb{H}^{n}\right) \subset L_{\rho, \theta_{1}}^{p, \kappa}\left(\mathbb{H}^{n}\right) \subset L_{\rho, \theta_{2}}^{p, \kappa}\left(\mathbb{H}^{n}\right) \\
W L^{p, \kappa}\left(\mathbb{H}^{n}\right) \subset W L_{\rho, \theta_{1}}^{p, \kappa}\left(\mathbb{H}^{n}\right) \subset W L_{\rho, \theta_{2}}^{p, \kappa}\left(\mathbb{H}^{n}\right),
\end{array}\right.
$$

for $0<\theta_{1}<\theta_{2}<\infty$. Hence $L^{p, \kappa}\left(\mathbb{H}^{n}\right) \subset L_{\rho, \infty}^{p, \kappa}\left(\mathbb{H}^{n}\right)$ and $W L^{p, \kappa}\left(\mathbb{H}^{n}\right) \subset W L_{\rho, \infty}^{p, \kappa}\left(\mathbb{H}^{n}\right)$ for $(p, \kappa) \in$ $[1, \infty) \times[0,1)$. The space $L_{\rho, \theta}^{p, \kappa}\left(\mathbb{H}^{n}\right)\left(\right.$ or $\left.W L_{\rho, \theta}^{p, \kappa}\left(\mathbb{H}^{n}\right)\right)$ could be viewed as an extension of Lebesgue (or weak Lebesgue) space on $\mathbb{H}^{n}$ (when $\kappa=\theta=0$ ). In this article we will extend the Hardy-Littlewood-Sobolev theorem on $\mathbb{H}^{n}$ to the Morrey spaces. We now present our main results.

Theorem 2.3 Let $0<\alpha<Q, 1<p<Q / \alpha$ and $1 / q=1 / p-\alpha / Q$. If $V \in R H_{s}$ with $s \geq Q / 2$ and $0<\kappa<p / q$, then the $\mathcal{L}$-fractional integral operator $\mathcal{I}_{\alpha}$ is bounded from $L_{\rho, \infty}^{p, \kappa}\left(\mathbb{H}^{n}\right)$ into $L_{\rho, \infty}^{q,(\kappa q) / p}\left(\mathbb{H}^{n}\right)$.

Theorem 2.4 Let $0<\alpha<Q, p=1$ and $q=Q /(Q-\alpha)$. If $V \in R H_{s}$ with $s \geq Q / 2$ and $0<\kappa<$ $1 / q$, then the $\mathcal{L}$-fractional integral operator $\mathcal{I}_{\alpha}$ is bounded from $L_{\rho, \infty}^{1, \kappa}\left(\mathbb{H}^{n}\right)$ into $W L_{\rho, \infty}^{q,(\kappa q)}\left(\mathbb{H}^{n}\right)$.

Before stating our next theorem, we need to introduce a new space $\mathrm{BMO}_{\rho, \infty}\left(\mathbb{H}^{n}\right)$ defined by

$$
\mathrm{BMO}_{\rho, \infty}\left(\mathbb{H}^{n}\right):=\bigcup_{\theta>0} \mathrm{BMO}_{\rho, \theta}\left(\mathbb{H}^{n}\right)
$$

where for $0<\theta<\infty$ the space $\mathrm{BMO}_{\rho, \theta}\left(\mathbb{H}^{n}\right)$ is defined to be the set of all locally integrable functions $f$ satisfying

$$
\frac{1}{\left|B\left(u_{0}, r\right)\right|} \int_{B\left(u_{0}, r\right)}\left|f(u)-f_{B\left(u_{0}, r\right)}\right| d u \leq C \cdot\left(1+\frac{r}{\rho\left(u_{0}\right)}\right)^{\theta},
$$

for all $u_{0} \in \mathbb{H}^{n}$ and $r>0, f_{B\left(u_{0}, r\right)}$ denotes the mean value of $f$ on $B\left(u_{0}, r\right)$, that is,

$$
f_{B\left(u_{0}, r\right)}:=\frac{1}{\left|B\left(u_{0}, r\right)\right|} \int_{B\left(u_{0}, r\right)} f(v) d v
$$


A norm for $f \in \mathrm{BMO}_{\rho, \theta}\left(\mathbb{H}^{n}\right)$, denoted by $\|f\|_{\mathrm{BMO}_{\rho, \theta}}$, is given by the infimum of the constants satisfying (2.2), or equivalently,

$$
\|f\|_{\mathrm{BMO}_{\rho, \theta}}:=\sup _{B\left(u_{0}, r\right)}\left(1+\frac{r}{\rho\left(u_{0}\right)}\right)^{-\theta}\left(\frac{1}{\left|B\left(u_{0}, r\right)\right|} \int_{B\left(u_{0}, r\right)}\left|f(u)-f_{B\left(u_{0}, r\right)}\right| d u\right),
$$

where the supremum is taken over all balls $B\left(u_{0}, r\right)$ with $u_{0} \in \mathbb{H}^{n}$ and $r>0$. Recall that in the setting of $\mathbb{R}^{n}$, the space $\mathrm{BMO}_{\rho, \theta}\left(\mathbb{R}^{n}\right)$ was first introduced by Bongioanni et al. [2] (see also [1]).

Moreover, given any $\beta \in[0,1]$, we introduce the space of Hölder continuous functions on $\mathbb{H}^{n}$, with exponent $\beta$.

$$
\mathcal{C}_{\rho, \infty}^{\beta}\left(\mathbb{H}^{n}\right):=\bigcup_{\theta>0} \mathcal{C}_{\rho, \theta}^{\beta}\left(\mathbb{H}^{n}\right),
$$

where for $0<\theta<\infty$ the space $\mathcal{C}_{\rho, \theta}^{\beta}\left(\mathbb{H}^{n}\right)$ is defined to be the set of all locally integrable functions $f$ satisfying

$$
\frac{1}{\left|B\left(u_{0}, r\right)\right|^{1+\beta / Q}} \int_{B\left(u_{0}, r\right)}\left|f(u)-f_{B\left(u_{0}, r\right)}\right| d u \leq C \cdot\left(1+\frac{r}{\rho\left(u_{0}\right)}\right)^{\theta},
$$

for all $u_{0} \in \mathbb{H}^{n}$ and $r \in(0, \infty)$. The smallest bound $C$ for which (2.3) is satisfied is then taken to be the norm of $f$ in this space and is denoted by $\|f\|_{\mathcal{C}_{\rho, \theta}^{\beta}}$. When $\theta=0$ or $V \equiv 0$, $\mathrm{BMO}_{\rho, \theta}\left(\mathbb{H}^{n}\right)$ and $\mathcal{C}_{\rho, \theta}^{\beta}\left(\mathbb{H}^{n}\right)$ will be simply written as $\mathrm{BMO}\left(\mathbb{H}^{n}\right)$ and $\mathcal{C}^{\beta}\left(\mathbb{H}^{n}\right)$, respectively. Note that when $\beta=0$ this space $\mathcal{C}_{\rho, \theta}^{\beta}\left(\mathbb{H}^{n}\right)$ reduces to the space $\mathrm{BMO}_{\rho, \theta}\left(\mathbb{H}^{n}\right)$ mentioned above.

For the case $\kappa \geq p / q$ of Theorem 2.3, we will prove the following result.

Theorem 2.5 Let $0<\alpha<Q, 1<p<Q / \alpha$ and $1 / q=1 / p-\alpha / Q$. If $V \in R H_{s}$ with $s \geq Q / 2$ and $p / q \leq \kappa<1$, then the $\mathcal{L}$-fractional integral operator $\mathcal{I}_{\alpha}$ is bounded from $L_{\rho, \infty}^{p, \kappa}\left(\mathbb{H}^{n}\right)$ into $\mathcal{C}_{\rho, \infty}^{\beta}\left(\mathbb{H}^{n}\right)$ with $\beta / Q=\kappa / p-1 / q$ and $\beta$ sufficiently small. To be more precise, $\beta<\delta \leq 1$ and $\delta$ is given as in Lemma 4.2 .

In particular, for the limiting case $\kappa=p / q$ (or $\beta=0$ ), we obtain the following result on BMO-type estimate of $\mathcal{I}_{\alpha}$.

Corollary 2.6 Let $0<\alpha<Q, 1<p<Q / \alpha$ and $1 / q=1 / p-\alpha / Q$. If $V \in R H_{s}$ with $s \geq Q / 2$ and $\kappa=p / q$, then the $\mathcal{L}$-fractional integral operator $\mathcal{I}_{\alpha}$ is bounded from $L_{\rho, \infty}^{p, \kappa}\left(\mathbb{H}^{n}\right)$ into $\mathrm{BMO}_{\rho, \infty}\left(\mathbb{H}^{n}\right)$.

\section{Proofs of Theorems 2.3 and 2.4}

In this section, we will prove the conclusions of Theorems 2.3 and 2.4. Let us recall that the $\mathcal{L}$-fractional integral operator of order $\alpha \in(0, Q)$ can be written as

$$
\mathcal{I}_{\alpha} f(u)=\mathcal{L}^{-\alpha / 2} f(u)=\int_{\mathbb{H}^{n}} \mathcal{K}_{\alpha}(u, v) f(v) d v,
$$

where

$$
\mathcal{K}_{\alpha}(u, v)=\frac{1}{\Gamma(\alpha / 2)} \int_{0}^{\infty} P_{s}(u, v) s^{\alpha / 2-1} d s .
$$


The following lemma gives the estimate of the kernel $\mathcal{K}_{\alpha}(u, v)$ related to the Schrödinger operator $\mathcal{L}$, which plays a key role in the proof of our main theorems.

Lemma 3.1 Let $V \in R H_{s}$ with $s \geq Q / 2$ and $0<\alpha<Q$. For every positive integer $N \geq 1$, there exists a positive constant $C_{N, \alpha}>0$ such that, for all $u$ and $v$ in $\mathbb{H}^{n}$,

$$
\left|\mathcal{K}_{\alpha}(u, v)\right| \leq C_{N, \alpha}\left(1+\frac{\left|v^{-1} u\right|}{\rho(u)}\right)^{-N} \frac{1}{\left|v^{-1} u\right|^{Q-\alpha}}
$$

Proof By Lemma 1.2 and (3.1), we have

$$
\begin{aligned}
\left|\mathcal{K}_{\alpha}(u, v)\right| & \leq \frac{1}{\Gamma(\alpha / 2)} \int_{0}^{\infty}\left|P_{s}(u, v)\right| s^{\alpha / 2-1} d s \\
& \leq \frac{1}{\Gamma(\alpha / 2)} \int_{0}^{\infty} \frac{C_{N}}{s^{Q / 2}} \cdot \exp \left(-\frac{\left|v^{-1} u\right|^{2}}{A s}\right)\left(1+\frac{\sqrt{s}}{\rho(u)}+\frac{\sqrt{s}}{\rho(v)}\right)^{-N} s^{\alpha / 2-1} d s \\
& \leq \frac{1}{\Gamma(\alpha / 2)} \int_{0}^{\infty} \frac{C_{N}}{s^{Q / 2}} \cdot \exp \left(-\frac{\left|v^{-1} u\right|^{2}}{A s}\right)\left(1+\frac{\sqrt{s}}{\rho(u)}\right)^{-N} s^{\alpha / 2-1} d s .
\end{aligned}
$$

We consider two cases $s>\left|v^{-1} u\right|^{2}$ and $0 \leq s \leq\left|v^{-1} u\right|^{2}$, respectively. Thus, $\left|\mathcal{K}_{\alpha}(u, v)\right| \leq I+I I$, where

$$
I=\frac{1}{\Gamma(\alpha / 2)} \int_{\left|v^{-1} u\right|^{2}}^{\infty} \frac{C_{N}}{s^{Q / 2}} \cdot \exp \left(-\frac{\left|v^{-1} u\right|^{2}}{A s}\right)\left(1+\frac{\sqrt{s}}{\rho(u)}\right)^{-N} s^{\alpha / 2-1} d s
$$

and

$$
I I=\frac{1}{\Gamma(\alpha / 2)} \int_{0}^{\left|v^{-1} u\right|^{2}} \frac{C_{N}}{s^{Q / 2}} \cdot \exp \left(-\frac{\left|v^{-1} u\right|^{2}}{A s}\right)\left(1+\frac{\sqrt{s}}{\rho(u)}\right)^{-N} s^{\alpha / 2-1} d s .
$$

When $s>\left|v^{-1} u\right|^{2}$, then $\sqrt{s}>\left|v^{-1} u\right|$, and hence

$$
\begin{aligned}
I & \leq \frac{1}{\Gamma(\alpha / 2)} \int_{\left|v^{-1} u\right|^{2}}^{\infty} \frac{C_{N}}{s^{Q / 2}} \cdot \exp \left(-\frac{\left|v^{-1} u\right|^{2}}{A s}\right)\left(1+\frac{\left|v^{-1} u\right|}{\rho(u)}\right)^{-N} s^{\alpha / 2-1} d s \\
& \leq C_{N, \alpha}\left(1+\frac{\left|v^{-1} u\right|}{\rho(u)}\right)^{-N} \int_{\left|v^{-1} u\right|^{2}}^{\infty} s^{\alpha / 2-Q / 2-1} d s \\
& \leq C_{N, \alpha}\left(1+\frac{\left|v^{-1} u\right|}{\rho(u)}\right)^{-N} \frac{1}{\left|v^{-1} u\right|^{Q-\alpha}},
\end{aligned}
$$

where the last integral converges because $0<\alpha<Q$. On the other hand,

$$
\begin{aligned}
I I & \leq C_{N, \alpha} \int_{0}^{\left|v^{-1} u\right|^{2}} \frac{1}{s^{Q / 2}} \cdot\left(\frac{\left|v^{-1} u\right|^{2}}{s}\right)^{-(Q / 2+N / 2)}\left(1+\frac{\sqrt{s}}{\rho(u)}\right)^{-N} s^{\alpha / 2-1} d s \\
& =C_{N, \alpha} \int_{0}^{\left|v^{-1} u\right|^{2}} \frac{1}{\left|v^{-1} u\right|^{Q}} \cdot\left(\frac{\sqrt{s}}{\left|v^{-1} u\right|}\right)^{N}\left(1+\frac{\sqrt{s}}{\rho(u)}\right)^{-N} s^{\alpha / 2-1} d s .
\end{aligned}
$$

It is easy to see that, when $0 \leq s \leq\left|v^{-1} u\right|^{2}$,

$$
\frac{\sqrt{s}}{\left|v^{-1} u\right|} \leq \frac{\sqrt{s}+\rho(u)}{\left|v^{-1} u\right|+\rho(u)}
$$


Hence,

$$
\begin{aligned}
I I & \leq C_{N, \alpha} \int_{0}^{\left|v^{-1} u\right|^{2}} \frac{1}{\left|v^{-1} u\right|^{Q}} \cdot\left(\frac{\sqrt{s}+\rho(u)}{\left|v^{-1} u\right|+\rho(u)}\right)^{N}\left(\frac{\sqrt{s}+\rho(u)}{\rho(u)}\right)^{-N} s^{\alpha / 2-1} d s \\
& =\frac{C_{N, \alpha}}{\left|v^{-1} u\right|^{Q}}\left(1+\frac{\left|v^{-1} u\right|}{\rho(u)}\right)^{-N} \int_{0}^{\left|v^{-1} u\right|^{2}} s^{\alpha / 2-1} d s \\
& =C_{N, \alpha}\left(1+\frac{\left|v^{-1} u\right|}{\rho(u)}\right)^{-N} \frac{1}{\left|v^{-1} u\right|^{Q-\alpha}} .
\end{aligned}
$$

Combining the estimates of $I$ and $I I$ yields the desired estimate (3.2) for $\alpha \in(0, Q)$. This concludes the proof of the lemma.

We are now ready to show our main theorems.

Proof of Theorem 2.3 By definition, we only need to show that, for any given ball $B=$ $B\left(u_{0}, r\right)$ of $\mathbb{H}^{n}$, there is some $\vartheta>0$ such that

$$
\left(\frac{1}{|B|^{\kappa q / p}} \int_{B}\left|\mathcal{I}_{\alpha} f(u)\right|^{q} d u\right)^{1 / q} \leq C \cdot\left(1+\frac{r}{\rho\left(u_{0}\right)}\right)^{\vartheta}
$$

holds for given $f \in L_{\rho, \infty}^{p, \kappa}\left(\mathbb{H}^{n}\right)$ with $(p, \kappa) \in(1, Q / \alpha) \times(0, p / q)$. Suppose that $f \in L_{\rho, \theta}^{p, \kappa}\left(\mathbb{H}^{n}\right)$ for some $\theta>0$. We decompose the function $f$ as

$$
\left\{\begin{array}{l}
f=f_{1}+f_{2} \in L_{\rho, \theta}^{p, \kappa}\left(\mathbb{H}^{n}\right) ; \\
f_{1}=f \cdot \chi_{2 B} ; \\
f_{2}=f \cdot \chi_{(2 B)},
\end{array}\right.
$$

where $2 B$ is the ball centered at $u_{0}$ of radius $2 r>0, \chi_{2 B}$ is the characteristic function of $2 B$ and $(2 B)^{c}=\mathbb{H}^{n} \backslash(2 B)$. Then, by the linearity of $\mathcal{I}_{\alpha}$, we write

$$
\begin{aligned}
\left(\frac{1}{|B|^{\kappa q / p}} \int_{B}\left|\mathcal{I}_{\alpha} f(u)\right|^{q} d u\right)^{1 / q} \leq & \left(\frac{1}{|B|^{\kappa q / p}} \int_{B}\left|\mathcal{I}_{\alpha} f_{1}(u)\right|^{q} d u\right)^{1 / q} \\
& +\left(\frac{1}{|B|^{\kappa q / p}} \int_{B}\left|\mathcal{I}_{\alpha} f_{2}(u)\right|^{q} d u\right)^{1 / q} \\
:= & I_{1}+I_{2} .
\end{aligned}
$$

In what follows, we consider each part separately. By Theorem 1.3 (1), we have

$$
\begin{aligned}
I_{1} & =\left(\frac{1}{|B|^{\kappa q / p}} \int_{B}\left|\mathcal{I}_{\alpha} f_{1}(u)\right|^{q} d u\right)^{1 / q} \\
& \leq C \cdot \frac{1}{|B|^{\kappa / p}}\left(\int_{\mathbb{H}^{n}}\left|f_{1}(u)\right|^{p} d u\right)^{1 / p} \\
& =C \cdot \frac{1}{|B|^{\kappa / p}}\left(\int_{2 B}|f(u)|^{p} d u\right)^{1 / p} \\
& \leq C\|f\|_{L_{\rho, \theta}^{p, \kappa}\left(\mathbb{H}^{n}\right)} \cdot \frac{|2 B|^{\kappa / p}}{|B|^{\kappa / p}} \cdot\left(1+\frac{2 r}{\rho\left(u_{0}\right)}\right)^{\theta} .
\end{aligned}
$$


Also observe that, for any fixed $\theta>0$,

$$
1 \leq\left(1+\frac{2 r}{\rho\left(u_{0}\right)}\right)^{\theta} \leq 2^{\theta}\left(1+\frac{r}{\rho\left(u_{0}\right)}\right)^{\theta}
$$

This in turn implies that

$$
I_{1} \leq C_{\theta, n}\|f\|_{L_{\rho, \theta}^{p, \kappa}\left(\mathbb{H}^{n}\right)}\left(1+\frac{r}{\rho\left(u_{0}\right)}\right)^{\theta} .
$$

Next we estimate the other term $I_{2}$. Notice that, for any $u \in B\left(u_{0}, r\right)$ and $v \in(2 B)^{c}$, one has

$$
\left|v^{-1} u\right|=\left|\left(v^{-1} u_{0}\right) \cdot\left(u_{0}^{-1} u\right)\right| \leq\left|v^{-1} u_{0}\right|+\left|u_{0}^{-1} u\right|
$$

and

$$
\left|v^{-1} u\right|=\left|\left(v^{-1} u_{0}\right) \cdot\left(u_{0}^{-1} u\right)\right| \geq\left|v^{-1} u_{0}\right|-\left|u_{0}^{-1} u\right|
$$

Thus,

$$
\frac{1}{2}\left|v^{-1} u_{0}\right| \leq\left|v^{-1} u\right| \leq \frac{3}{2}\left|v^{-1} u_{0}\right|
$$

i.e., $\left|v^{-1} u\right| \approx\left|v^{-1} u_{0}\right|$. It then follows from Lemma 3.1 that, for any $u \in B\left(u_{0}, r\right)$ and any positive integer $N$,

$$
\begin{aligned}
\left|\mathcal{I}_{\alpha} f_{2}(u)\right| & \leq \int_{(2 B)^{c}}\left|\mathcal{K}_{\alpha}(u, v)\right| \cdot|f(v)| d v \\
& \leq C_{N, \alpha} \int_{(2 B)^{c}}\left(1+\frac{\left|v^{-1} u\right|}{\rho(u)}\right)^{-N} \frac{1}{\left|v^{-1} u\right|^{Q-\alpha}} \cdot|f(v)| d v \\
& \leq C_{N, \alpha, n} \int_{(2 B)^{c}}\left(1+\frac{\left|v^{-1} u_{0}\right|}{\rho(u)}\right)^{-N} \frac{1}{\left|v^{-1} u_{0}\right|^{Q-\alpha}} \cdot|f(v)| d v \\
& =C_{N, \alpha, n} \sum_{k=1}^{\infty} \int_{2^{k} r \leq\left|v^{-1} u_{0}\right|<2^{k+1} r}\left(1+\frac{\left|v^{-1} u_{0}\right|}{\rho(u)}\right)^{-N} \frac{1}{\left|v^{-1} u_{0}\right|^{Q-\alpha}} \cdot|f(v)| d v \\
& \leq C_{N, \alpha, n} \sum_{k=1}^{\infty} \frac{1}{\left|B\left(u_{0}, 2^{k+1} r\right)\right|^{1-(\alpha / Q)}} \int_{\left|v^{-1} u_{0}\right|<2^{k+1} r}\left(1+\frac{2^{k} r}{\rho(u)}\right)^{-N}|f(v)| d v .
\end{aligned}
$$

In view of (1.4) and (3.4), we can further obtain

$$
\begin{aligned}
\left|\mathcal{I}_{\alpha} f_{2}(u)\right| \leq & C \sum_{k=1}^{\infty} \frac{1}{\left|B\left(u_{0}, 2^{k+1} r\right)\right|^{1-(\alpha / Q)}} \\
& \times \int_{\left|v^{-1} u_{0}\right|<2^{k+1} r}\left(1+\frac{r}{\rho\left(u_{0}\right)}\right)^{N \cdot \frac{N_{0}}{N_{0}+1}}\left(1+\frac{2^{k} r}{\rho\left(u_{0}\right)}\right)^{-N}|f(v)| d v
\end{aligned}
$$




$$
\begin{aligned}
\leq & C \sum_{k=1}^{\infty} \frac{1}{\left|B\left(u_{0}, 2^{k+1} r\right)\right|^{1-(\alpha / Q)}} \\
& \times \int_{B\left(u_{0}, 2^{k+1} r\right)}\left(1+\frac{r}{\rho\left(u_{0}\right)}\right)^{N \cdot \frac{N_{0}}{N_{0}+1}}\left(1+\frac{2^{k+1} r}{\rho\left(u_{0}\right)}\right)^{-N}|f(v)| d v .
\end{aligned}
$$

We consider each term in the sum of (3.6) separately. By using Hölder's inequality, we find that, for each integer $k \geq 1$,

$$
\begin{aligned}
& \frac{1}{\left|B\left(u_{0}, 2^{k+1} r\right)\right|^{1-(\alpha / Q)}} \int_{B\left(u_{0}, 2^{k+1} r\right)}|f(v)| d v \\
& \leq \frac{1}{\left|B\left(u_{0}, 2^{k+1} r\right)\right|^{1-(\alpha / Q)}}\left(\int_{B\left(u_{0}, 2^{k+1} r\right)}|f(v)|^{p} d v\right)^{1 / p}\left(\int_{B\left(u_{0}, 2^{k+1} r\right)} 1 d v\right)^{1 / p^{\prime}} \\
& \quad \leq C\|f\|_{L_{\rho, \theta}^{p, \kappa}\left(\mathbb{H}^{n}\right)} \cdot \frac{\left|B\left(u_{0}, 2^{k+1} r\right)\right|^{\kappa / p}}{\left|B\left(u_{0}, 2^{k+1} r\right)\right|^{1 / q}}\left(1+\frac{2^{k+1} r}{\rho\left(u_{0}\right)}\right)^{\theta}
\end{aligned}
$$

This allows us to obtain

$$
\begin{aligned}
I_{2} \leq & C\|f\|_{L_{\rho, \theta}^{p, \kappa}\left(\mathbb{H}^{n}\right)} \cdot \frac{\left|B\left(u_{0}, r\right)\right|^{1 / q}}{\left|B\left(u_{0}, r\right)\right|^{\kappa / p}} \sum_{k=1}^{\infty} \frac{\left|B\left(u_{0}, 2^{k+1} r\right)\right|^{\kappa / p}}{\left|B\left(u_{0}, 2^{k+1} r\right)\right|^{1 / q}} \\
& \times\left(1+\frac{r}{\rho\left(u_{0}\right)}\right)^{N \cdot \frac{N_{0}}{N_{0}+1}}\left(1+\frac{2^{k+1} r}{\rho\left(u_{0}\right)}\right)^{-N+\theta} \\
= & C\|f\|_{L_{\rho, \theta}^{p, \kappa}\left(\mathbb{H}^{n}\right)}\left(1+\frac{r}{\rho\left(u_{0}\right)}\right)^{N \cdot \frac{N_{0}}{N_{0}+1}} \sum_{k=1}^{\infty} \frac{\left|B\left(u_{0}, r\right)\right|^{1 / q-\kappa / p}}{\left|B\left(u_{0}, 2^{k+1} r\right)\right|^{1 / q-\kappa / p}}\left(1+\frac{2^{k+1} r}{\rho\left(u_{0}\right)}\right)^{-N+\theta} .
\end{aligned}
$$

Thus, by choosing $N$ large enough so that $N>\theta$, and the last series is convergent, then we have

$$
\begin{aligned}
I_{2} & \leq C\|f\|_{L_{\rho, \theta}^{p, k}\left(\mathbb{H}^{n}\right)}\left(1+\frac{r}{\rho\left(u_{0}\right)}\right)^{N \cdot \frac{N_{0}}{N_{0}+1}} \sum_{k=1}^{\infty}\left(\frac{\left|B\left(u_{0}, r\right)\right|}{\left|B\left(u_{0}, 2^{k+1} r\right)\right|}\right)^{(1 / q-\kappa / p)} \\
& \leq C\|f\|_{L_{\rho, \theta}^{p, \kappa}\left(\mathbb{H}^{n}\right)}\left(1+\frac{r}{\rho\left(u_{0}\right)}\right)^{N \cdot \frac{N_{0}}{N_{0}+1}},
\end{aligned}
$$

where the last inequality follows from the fact that $1 / q-\kappa / p>0$. Summing up the above estimates for $I_{1}$ and $I_{2}$ and letting $\vartheta=\max \left\{\theta, N \cdot \frac{N_{0}}{N_{0}+1}\right\}$, we obtain the desired inequality (3.3). This completes the proof of Theorem 2.3.

Proof of Theorem 2.4 To prove Theorem 2.4, by definition, it suffices to prove that, for each given ball $B=B\left(u_{0}, r\right)$ of $\mathbb{H}^{n}$, there is some $\vartheta>0$ such that

$$
\frac{1}{|B|^{\kappa}} \sup _{\lambda>0} \lambda \cdot\left|\left\{u \in B:\left|\mathcal{I}_{\alpha} f(u)\right|>\lambda\right\}\right|^{1 / q} \leq C \cdot\left(1+\frac{r}{\rho\left(u_{0}\right)}\right)^{\vartheta}
$$


holds for given $f \in L_{\rho, \infty}^{1, \kappa}\left(\mathbb{H}^{n}\right)$ with $0<\kappa<1 / q$ and $q=Q /(Q-\alpha)$. Now suppose that $f \in$ $L_{\rho, \theta}^{1, \kappa}\left(\mathbb{H}^{n}\right)$ for some $\theta>0$. We decompose the function $f$ as

$$
\left\{\begin{array}{l}
f=f_{1}+f_{2} \in L_{\rho, \theta}^{1, \kappa}\left(\mathbb{H}^{n}\right) \\
f_{1}=f \cdot \chi_{2 B} ; \\
f_{2}=f \cdot \chi_{(2 B)} \cdot
\end{array}\right.
$$

Then, for any given $\lambda>0$, by the linearity of $\mathcal{I}_{\alpha}$, we can write

$$
\begin{aligned}
& \frac{1}{|B|^{\kappa}} \lambda \cdot\left|\left\{u \in B:\left|\mathcal{I}_{\alpha} f(u)\right|>\lambda\right\}\right|^{1 / q} \\
& \leq \frac{1}{|B|^{\kappa}} \lambda \cdot\left|\left\{u \in B:\left|\mathcal{I}_{\alpha} f_{1}(u)\right|>\lambda / 2\right\}\right|^{1 / q} \\
& \quad+\frac{1}{|B|^{\kappa}} \lambda \cdot\left|\left\{u \in B:\left|\mathcal{I}_{\alpha} f_{2}(u)\right|>\lambda / 2\right\}\right|^{1 / q} \\
& :=J_{1}+J_{2} .
\end{aligned}
$$

We first give the estimate for the term $J_{1}$. By Theorem 1.3 (2), we get

$$
\begin{aligned}
J_{1} & =\frac{1}{|B|^{\kappa}} \lambda \cdot\left|\left\{u \in B:\left|\mathcal{I}_{\alpha} f_{1}(u)\right|>\lambda / 2\right\}\right|^{1 / q} \\
& \leq C \cdot \frac{1}{|B|^{\kappa}}\left(\int_{\mathbb{H}^{n}}\left|f_{1}(u)\right| d u\right) \\
& =C \cdot \frac{1}{|B|^{\kappa}}\left(\int_{2 B}|f(u)| d u\right) \\
& \leq C\|f\|_{L_{\rho, \theta}^{1, \kappa}\left(\mathbb{H}^{n}\right)} \cdot \frac{|2 B|^{\kappa}}{|B|^{\kappa}}\left(1+\frac{2 r}{\rho\left(u_{0}\right)}\right)^{\theta} .
\end{aligned}
$$

Therefore, in view of (3.4),

$$
J_{1} \leq C\|f\|_{L_{\rho, \theta}^{1, \kappa}\left(\mathbb{H}^{n}\right)} \cdot\left(1+\frac{r}{\rho\left(u_{0}\right)}\right)^{\theta} .
$$

As for the second term $J_{2}$, by using the pointwise inequality (3.6) and Chebyshev's inequality, we can deduce that

$$
\begin{aligned}
J_{2}= & \frac{1}{|B|^{\kappa}} \lambda \cdot\left|\left\{u \in B:\left|\mathcal{I}_{\alpha} f_{2}(u)\right|>\lambda / 2\right\}\right|^{1 / q} \\
\leq & \frac{2}{|B|^{\kappa}}\left(\int_{B}\left|\mathcal{I}_{\alpha} f_{2}(u)\right|^{q} d u\right)^{1 / q} \\
\leq & C \cdot \frac{|B|^{1 / q}}{|B|^{\kappa}} \sum_{k=1}^{\infty} \frac{1}{\left|B\left(u_{0}, 2^{k+1} r\right)\right|^{1-(\alpha / Q)}} \\
& \times \int_{B\left(u_{0}, 2^{k+1} r\right)}\left(1+\frac{r}{\rho\left(u_{0}\right)}\right)^{N \cdot \frac{N_{0}}{N_{0}+1}}\left(1+\frac{2^{k+1} r}{\rho\left(u_{0}\right)}\right)^{-N}|f(v)| d v .
\end{aligned}
$$


We consider each term in the sum of (3.8) separately. For each integer $k \geq 1$, we compute

$$
\begin{aligned}
& \frac{1}{\left|B\left(u_{0}, 2^{k+1} r\right)\right|^{1-(\alpha / Q)}} \int_{B\left(u_{0}, 2^{k+1} r\right)}|f(v)| d v \\
& \quad \leq C\|f\|_{L_{\rho, \theta}^{1, \kappa}\left(\mathbb{H}^{n}\right)} \cdot \frac{\left|B\left(u_{0}, 2^{k+1} r\right)\right|^{\kappa}}{\left|B\left(u_{0}, 2^{k+1} r\right)\right|^{1 / q}}\left(1+\frac{2^{k+1} r}{\rho\left(u_{0}\right)}\right)^{\theta} .
\end{aligned}
$$

Consequently,

$$
\begin{aligned}
& J_{2} \leq C\|f\|_{L_{\rho, \theta}^{1, \kappa}\left(\mathbb{H}^{n}\right)} \cdot \frac{\left|B\left(u_{0}, r\right)\right|^{1 / q}}{\left|B\left(u_{0}, r\right)\right|^{\kappa}} \sum_{k=1}^{\infty} \frac{\left|B\left(u_{0}, 2^{k+1} r\right)\right|^{\kappa}}{\left|B\left(u_{0}, 2^{k+1} r\right)\right|^{1 / q}} \\
& \times\left(1+\frac{r}{\rho\left(u_{0}\right)}\right)^{N \cdot \frac{N_{0}}{N_{0}+1}}\left(1+\frac{2^{k+1} r}{\rho\left(u_{0}\right)}\right)^{-N+\theta} \\
& =C\|f\|_{L_{\rho, \theta}^{1, \kappa}\left(\mathbb{H}^{n}\right)}\left(1+\frac{r}{\rho\left(u_{0}\right)}\right)^{N \cdot N_{0}} \sum_{k=1}^{N_{0}+1} \frac{\left|B\left(u_{0}, r\right)\right|^{1 / q-\kappa}}{\left|B\left(u_{0}, 2^{k+1} r\right)\right|^{1 / q-\kappa}}\left(1+\frac{2^{k+1} r}{\rho\left(u_{0}\right)}\right)^{-N+\theta} .
\end{aligned}
$$

Therefore, by selecting $N$ large enough so that $N>\theta$, we thus have

$$
\begin{aligned}
& J_{2} \leq C\|f\|_{L_{\rho, \theta}^{1, \kappa}\left(\mathbb{H}^{n}\right)}\left(1+\frac{r}{\rho\left(u_{0}\right)}\right)^{N \cdot N_{0}} \sum_{k=1}^{N_{0}}\left(\frac{\left|B\left(u_{0}, r\right)\right|}{\left|B\left(u_{0}, 2^{k+1} r\right)\right|}\right)^{(1 / q-\kappa)} \\
& \leq C\|f\|_{L_{\rho, \theta}^{1, \kappa}\left(\mathbb{H}^{n}\right)}\left(1+\frac{r}{\rho\left(u_{0}\right)}\right)^{N \cdot \frac{N_{0}}{N_{0}+1}},
\end{aligned}
$$

where the last step is due to the fact that $0<\kappa<1 / q$. Let $\vartheta=\max \left\{\theta, N \cdot \frac{N_{0}}{N_{0}+1}\right\}$. Here $N$ is an appropriate constant. Summing up the above estimates for $J_{1}$ and $J_{2}$, and then taking the supremum over all $\lambda>0$, we obtain the desired inequality (3.7). This finishes the proof of Theorem 2.4.

\section{Proof of Theorem 2.5}

We need the following lemma which establishes the Lipschitz regularity of the kernel $P_{s}(u, v)$. See Lemma 11 and Remark 4 in [8].

Lemma 4.1 ([8]) Let $V \in R H_{s}$ with $s \geq Q / 2$. For every positive integer $N \geq 1$, there exists a positive constant $C_{N}>0$ such that, for all $u$ and $v$ in $\mathbb{H}^{n}$, and for some fixed $0<\delta \leq 1$,

$$
\left|P_{s}(u \cdot h, v)-P_{s}(u, v)\right| \leq C_{N}\left(\frac{|h|}{\sqrt{s}}\right)^{\delta} s^{-Q / 2} \exp \left(-\frac{\left|v^{-1} u\right|^{2}}{A s}\right)\left(1+\frac{\sqrt{s}}{\rho(u)}+\frac{\sqrt{s}}{\rho(v)}\right)^{-N}
$$

whenever $|h| \leq\left|v^{-1} u\right| / 2$.

Based on the above lemma, we are able to prove the following result, which plays a key role in the proof of our main theorem.

Lemma 4.2 Let $V \in R H_{s}$ with $s \geq Q / 2$ and $0<\alpha<Q$. For every positive integer $N \geq 1$, there exists a positive constant $C_{N, \alpha}>0$ such that, for all $u, v$ and $w$ in $\mathbb{H}^{n}$, and for some 
Wang Journal of Inequalities and Applications

(2019) 2019:232

Page 16 of 21

fixed $0<\delta \leq 1$,

$$
\left|\mathcal{K}_{\alpha}(u, w)-\mathcal{K}_{\alpha}(v, w)\right| \leq C_{N, \alpha}\left(1+\frac{\left|w^{-1} u\right|}{\rho(u)}\right)^{-N} \frac{\left|v^{-1} u\right|^{\delta}}{\left|w^{-1} u\right|^{Q-\alpha+\delta}}
$$

whenever $\left|v^{-1} u\right| \leq\left|w^{-1} u\right| / 2$.

Proof In view of Lemma 4.1 and (3.1), we have

$$
\begin{aligned}
&\left|\mathcal{K}_{\alpha}(u, w)-\mathcal{K}_{\alpha}(v, w)\right| \\
&=\frac{1}{\Gamma(\alpha / 2)}\left|\int_{0}^{\infty} P_{s}(u, w) s^{\alpha / 2-1} d s-\int_{0}^{\infty} P_{s}(v, w) s^{\alpha / 2-1} d s\right| \\
& \leq \frac{1}{\Gamma(\alpha / 2)} \int_{0}^{\infty}\left|P_{s}\left(u \cdot\left(u^{-1} v\right), w\right)-P_{s}(u, w)\right| s^{\alpha / 2-1} d s \\
& \leq \frac{1}{\Gamma(\alpha / 2)} \int_{0}^{\infty} C_{N} \cdot\left(\frac{\left|u^{-1} v\right|}{\sqrt{s}}\right)^{\delta} s^{-Q / 2} \\
& \quad \times \exp \left(-\frac{\left|w^{-1} u\right|^{2}}{A s}\right)\left(1+\frac{\sqrt{s}}{\rho(u)}+\frac{\sqrt{s}}{\rho(w)}\right)^{-N} s^{\alpha / 2-1} d s \\
& \leq \frac{1}{\Gamma(\alpha / 2)} \int_{0}^{\infty} C_{N} \cdot\left(\frac{\left|u^{-1} v\right|}{\sqrt{s}}\right)^{\delta} s^{-Q / 2} \exp \left(-\frac{\left|w^{-1} u\right|^{2}}{A s}\right)\left(1+\frac{\sqrt{s}}{\rho(u)}\right)^{-N} s^{\alpha / 2-1} d s .
\end{aligned}
$$

Arguing as in the proof of Lemma 3.1, consider two cases as below: $s>\left|w^{-1} u\right|^{2}$ and $0 \leq$ $s \leq\left|w^{-1} u\right|^{2}$. Then the right-hand side of the above expression can be written as $I I I+I V$, where

$$
I I I=\frac{1}{\Gamma(\alpha / 2)} \int_{\left|w^{-1} u\right|^{2}}^{\infty} \frac{C_{N}}{s^{Q / 2}} \cdot\left(\frac{\left|u^{-1} v\right|}{\sqrt{s}}\right)^{\delta} \exp \left(-\frac{\left|w^{-1} u\right|^{2}}{A s}\right)\left(1+\frac{\sqrt{s}}{\rho(u)}\right)^{-N} s^{\alpha / 2-1} d s
$$

and

$$
I V=\frac{1}{\Gamma(\alpha / 2)} \int_{0}^{\left|w^{-1} u\right|^{2}} \frac{C_{N}}{s^{Q / 2}} \cdot\left(\frac{\left|u^{-1} v\right|}{\sqrt{s}}\right)^{\delta} \exp \left(-\frac{\left|w^{-1} u\right|^{2}}{A s}\right)\left(1+\frac{\sqrt{s}}{\rho(u)}\right)^{-N} s^{\alpha / 2-1} d s
$$

When $s>\left|w^{-1} u\right|^{2}$, then $\sqrt{s}>\left|w^{-1} u\right|$, and hence

$$
\begin{aligned}
I I I & \leq \frac{1}{\Gamma(\alpha / 2)} \int_{\left|w^{-1} u\right|^{2}}^{\infty} \frac{C_{N}}{s^{Q / 2}} \cdot\left(\frac{\left|u^{-1} v\right|}{\left|w^{-1} u\right|}\right)^{\delta} \exp \left(-\frac{\left|w^{-1} u\right|^{2}}{A s}\right)\left(1+\frac{\left|w^{-1} u\right|}{\rho(u)}\right)^{-N} s^{\alpha / 2-1} d s \\
& \leq C_{N, \alpha}\left(1+\frac{\left|w^{-1} u\right|}{\rho(u)}\right)^{-N}\left(\frac{\left|u^{-1} v\right|}{\left|w^{-1} u\right|}\right)^{\delta} \int_{\left|w^{-1} u\right|^{2}}^{\infty} s^{\alpha / 2-Q / 2-1} d s \\
& =C_{N, \alpha}\left(1+\frac{\left|w^{-1} u\right|}{\rho(u)}\right)^{-N} \frac{\left|v^{-1} u\right|^{\delta}}{\left|w^{-1} u\right|^{Q-\alpha+\delta}},
\end{aligned}
$$


where the last equality holds since $\left|u^{-1} v\right|=\left|v^{-1} u\right|$ and $0<\alpha<Q$. On the other hand,

$$
\begin{aligned}
I V & \leq C_{N, \alpha} \int_{0}^{\left|w^{-1} u\right|^{2}} \frac{1}{s^{Q / 2}} \cdot\left(\frac{\left|u^{-1} v\right|}{\sqrt{s}}\right)^{\delta}\left(\frac{\left|w^{-1} u\right|^{2}}{s}\right)^{-(Q / 2+N / 2+\delta / 2)}\left(1+\frac{\sqrt{s}}{\rho(u)}\right)^{-N} s^{\alpha / 2-1} d s \\
& =C_{N, \alpha} \int_{0}^{\left|w^{-1} u\right|^{2}} \frac{\left|u^{-1} v\right|^{\delta}}{\left|w^{-1} u\right|^{Q+\delta}}\left(\frac{\sqrt{s}}{\left|w^{-1} u\right|}\right)^{N}\left(1+\frac{\sqrt{s}}{\rho(u)}\right)^{-N} s^{\alpha / 2-1} d s .
\end{aligned}
$$

It is easy to check that when $0 \leq s \leq\left|w^{-1} u\right|^{2}$,

$$
\frac{\sqrt{s}}{\left|w^{-1} u\right|} \leq \frac{\sqrt{s}+\rho(u)}{\left|w^{-1} u\right|+\rho(u)} .
$$

This in turn implies that

$$
\begin{aligned}
I V & \leq C_{N, \alpha} \int_{0}^{\left|w^{-1} u\right|^{2}} \frac{\left|u^{-1} v\right|^{\delta}}{\left|w^{-1} u\right|^{Q+\delta}}\left(\frac{\sqrt{s}+\rho(u)}{\left|w^{-1} u\right|+\rho(u)}\right)^{N}\left(\frac{\sqrt{s}+\rho(u)}{\rho(u)}\right)^{-N} s^{\alpha / 2-1} d s \\
& =C_{N, \alpha} \cdot \frac{\left|u^{-1} v\right|^{\delta}}{\left|w^{-1} u\right|^{Q+\delta}}\left(1+\frac{\left|w^{-1} u\right|}{\rho(u)}\right)^{-N} \int_{0}^{\left|w^{-1} u\right|^{2}} s^{\alpha / 2-1} d s \\
& =C_{N, \alpha}\left(1+\frac{\left|w^{-1} u\right|}{\rho(u)}\right)^{-N} \frac{\left|v^{-1} u\right|^{\delta}}{\left|w^{-1} u\right|^{Q-\alpha+\delta}},
\end{aligned}
$$

where the last step holds because $\left|u^{-1} v\right|=\left|v^{-1} u\right|$. Combining the estimates of III and $I V$ produces the desired inequality (4.1) for $\alpha \in(0, Q)$. This concludes the proof of the lemma.

We are now in a position to give the proof of Theorem 2.5.

Proof of Theorem 2.5 Fix a ball $B=B\left(u_{0}, r\right)$ with $u_{0} \in \mathbb{H}^{n}$ and $r \in(0, \infty)$, it suffices to prove that the inequality

$$
\frac{1}{|B|^{1+\beta / Q}} \int_{B}\left|\mathcal{I}_{\alpha} f(u)-\left(\mathcal{I}_{\alpha} f\right)_{B}\right| d u \leq C \cdot\left(1+\frac{r}{\rho\left(u_{0}\right)}\right)^{\vartheta}
$$

holds for given $f \in L_{\rho, \infty}^{p, \kappa}\left(\mathbb{H}^{n}\right)$ with $1<p<q<\infty$ and $p / q \leq \kappa<1$, where $0<\alpha<Q$ and $\left(\mathcal{I}_{\alpha} f\right)_{B}$ denotes the average of $\mathcal{I}_{\alpha} f$ over $B$. Suppose that $f \in L_{\rho, \theta}^{p, \kappa}\left(\mathbb{H}^{n}\right)$ for some $\theta>0$. Decompose the function $f$ as $f=f_{1}+f_{2}$, where $f_{1}=f \cdot \chi_{4 B}, f_{2}=f \cdot \chi_{(4 B)^{c}}, 4 B=B\left(u_{0}, 4 r\right)$ and $(4 B)^{c}=\mathbb{H}^{n} \backslash(4 B)$. By the linearity of the $\mathcal{L}$-fractional integral operator $\mathcal{I}_{\alpha}$, the left-hand side of (4.2) can be written as

$$
\begin{aligned}
& \frac{1}{|B|^{1+\beta / Q}} \int_{B}\left|\mathcal{I}_{\alpha} f(u)-\left(\mathcal{I}_{\alpha} f\right)_{B}\right| d u \\
& \quad \leq \frac{1}{|B|^{1+\beta / Q}} \int_{B}\left|\mathcal{I}_{\alpha} f_{1}(u)-\left(\mathcal{I}_{\alpha} f_{1}\right)_{B}\right| d u+\frac{1}{|B|^{1+\beta / Q}} \int_{B}\left|\mathcal{I}_{\alpha} f_{2}(u)-\left(\mathcal{I}_{\alpha} f_{2}\right)_{B}\right| d u \\
& \quad:=K_{1}+K_{2} .
\end{aligned}
$$


Wang Journal of Inequalities and Applications

(2019) 2019:232

Page 18 of 21

Let us consider the first term $K_{1}$. Applying the strong-type $(p, q)$ estimate of $\mathcal{I}_{\alpha}$ (see Theorem 1.3) and Hölder's inequality, we obtain

$$
\begin{aligned}
K_{1} & \leq \frac{2}{|B|^{1+\beta / Q}} \int_{B}\left|\mathcal{I}_{\alpha} f_{1}(u)\right| d u \\
& \leq \frac{2}{|B|^{1+\beta / Q}}\left(\int_{B}\left|\mathcal{I}_{\alpha} f_{1}(u)\right|^{q} d u\right)^{1 / q}\left(\int_{B} 1 d u\right)^{1 / q^{\prime}} \\
& \leq \frac{C}{|B|^{1+\beta / Q}}\left(\int_{4 B}|f(u)|^{p} d u\right)^{1 / p}|B|^{1 / q^{\prime}} \\
& \leq C\|f\|_{L_{\rho, \theta}^{p, \kappa}\left(\mathbb{H}^{n}\right)} \cdot \frac{\left|B\left(u_{0}, 4 r\right)\right|^{\kappa / p}}{\left|B\left(u_{0}, r\right)\right|^{1 / q+\beta / Q}}\left(1+\frac{4 r}{\rho\left(u_{0}\right)}\right)^{\theta} .
\end{aligned}
$$

Using the inequalities (1.1) and (3.4), and noting the fact that $\beta / Q=\kappa / p-1 / q$, we derive

$$
\begin{aligned}
K_{1} & \leq C_{n}\|f\|_{L_{\rho, \theta}^{p, \kappa}\left(\mathbb{H}^{n}\right)}\left(1+\frac{4 r}{\rho\left(u_{0}\right)}\right)^{\theta} \\
& \leq C_{n, \theta}\|f\|_{L_{\rho, \theta}^{p, \kappa}\left(\mathbb{H}^{n}\right)}\left(1+\frac{r}{\rho\left(u_{0}\right)}\right)^{\theta} .
\end{aligned}
$$

Let us now turn to the estimate of the second term $K_{2}$. For any $u \in B\left(u_{0}, r\right)$,

$$
\begin{aligned}
\left|\mathcal{I}_{\alpha} f_{2}(u)-\left(\mathcal{I}_{\alpha} f_{2}\right)_{B}\right| & =\left|\frac{1}{|B|} \int_{B}\left[\mathcal{I}_{\alpha} f_{2}(u)-\mathcal{I}_{\alpha} f_{2}(v)\right] d v\right| \\
& =\left|\frac{1}{|B|} \int_{B}\left\{\int_{(4 B)^{c}}\left[\mathcal{K}_{\alpha}(u, w)-\mathcal{K}_{\alpha}(v, w)\right] f(w) d w\right\} d v\right| \\
& \leq \frac{1}{|B|} \int_{B}\left\{\int_{(4 B)^{c}}\left|\mathcal{K}_{\alpha}(u, w)-\mathcal{K}_{\alpha}(v, w)\right| \cdot|f(w)| d w\right\} d v .
\end{aligned}
$$

By using the same arguments as for Theorem 2.3, we find that

$$
\left|v^{-1} u\right| \leq\left|w^{-1} u\right| / 2 \text { and }\left|w^{-1} u\right| \approx\left|w^{-1} u_{0}\right|
$$

whenever $u, v \in B$ and $w \in(4 B)^{c}$. This fact along with Lemma 4.2 yields

$$
\begin{aligned}
& \left|\mathcal{I}_{\alpha} f_{2}(u)-\left(\mathcal{I}_{\alpha} f_{2}\right)_{B}\right| \\
& \quad \leq \frac{C_{N, \alpha}}{|B|} \int_{B}\left\{\int_{(4 B)^{c}}\left(1+\frac{\left|w^{-1} u\right|}{\rho(u)}\right)^{-N} \frac{\left|v^{-1} u\right|^{\delta}}{\left|w^{-1} u\right|^{Q-\alpha+\delta}} \cdot|f(w)| d w\right\} d v \\
& \quad \leq C_{N, \alpha, n} \int_{(4 B)^{c}}\left(1+\frac{\left|w^{-1} u_{0}\right|}{\rho(u)}\right)^{-N} \frac{r^{\delta}}{\left|w^{-1} u_{0}\right|^{Q-\alpha+\delta}} \cdot|f(w)| d w \\
& \quad=C_{N, \alpha, n} \sum_{k=2}^{\infty} \int_{2^{k} r \leq\left|w^{-1} u_{0}\right|<2^{k+1} r}\left(1+\frac{\left|w^{-1} u_{0}\right|}{\rho(u)}\right)^{-N} \frac{r^{\delta}}{\left|w^{-1} u_{0}\right|^{Q-\alpha+\delta}} \cdot|f(w)| d w \\
& \quad \leq C_{N, \alpha, n} \sum_{k=2}^{\infty} \frac{1}{2^{k \delta}} \cdot \frac{1}{\left|B\left(u_{0}, 2^{k+1} r\right)\right|^{1-(\alpha / Q)}} \int_{B\left(u_{0}, 2^{k+1} r\right)}\left(1+\frac{2^{k} r}{\rho(u)}\right)^{-N}|f(w)| d w .
\end{aligned}
$$


Furthermore, by using Hölder's inequality and (1.4), we deduce that, for any $u \in B\left(u_{0}, r\right)$,

$$
\begin{aligned}
\left|\mathcal{I}_{\alpha} f_{2}(u)-\left(\mathcal{I}_{\alpha} f_{2}\right)_{B}\right| & \\
\leq & C \sum_{k=2}^{\infty} \frac{1}{2^{k \delta}} \cdot \frac{1}{\left|B\left(u_{0}, 2^{k+1} r\right)\right|^{1-(\alpha / Q)}}\left(1+\frac{r}{\rho\left(u_{0}\right)}\right)^{N \cdot \frac{N_{0}}{N_{0}+1}}\left(1+\frac{2^{k+1} r}{\rho\left(u_{0}\right)}\right)^{-N} \\
& \times\left(\int_{B\left(u_{0}, 2^{k+1} r\right)}|f(w)|^{p} d w\right)^{1 / p}\left(\int_{B\left(u_{0}, 2^{k+1} r\right)} 1 d w\right)^{1 / p^{\prime}} \\
\leq & C\|f\|_{L_{\rho, \theta}^{p, \kappa}\left(\mathbb{H}^{n}\right)} \sum_{k=2}^{\infty} \frac{1}{2^{k \delta}} \cdot\left(1+\frac{r}{\rho\left(u_{0}\right)}\right)^{N \cdot \frac{N_{0}}{N_{0}+1}}\left(1+\frac{2^{k+1} r}{\rho\left(u_{0}\right)}\right)^{-N} \\
& \times \frac{\left|B\left(u_{0}, 2^{k+1} r\right)\right|^{\kappa / p}}{\left|B\left(u_{0}, 2^{k+1} r\right)\right|^{1 / q}}\left(1+\frac{2^{k+1} r}{\rho\left(u_{0}\right)}\right)^{\theta} \\
= & C\|f\|_{L_{\rho, \theta}^{p, \kappa}\left(\mathbb{H}^{n}\right)} \sum_{k=2}^{\infty} \frac{\left|B\left(u_{0}, 2^{k+1} r\right)\right|^{\beta / Q}}{2^{k \delta}} \cdot\left(1+\frac{r}{\rho\left(u_{0}\right)}\right)^{N \cdot \frac{N_{0}}{N_{0}+1}}\left(1+\frac{2^{k+1} r}{\rho\left(u_{0}\right)}\right)^{-N+\theta},
\end{aligned}
$$

where the last equality is due to the assumption $\beta / Q=\kappa / p-1 / q$. From the pointwise estimate (4.4) and (1.1), it readily follows that

$$
\begin{aligned}
K_{2} & =\frac{1}{|B|^{1+\beta / Q}} \int_{B}\left|\mathcal{I}_{\alpha} f_{2}(u)-\left(\mathcal{I}_{\alpha} f_{2}\right)_{B}\right| d u \\
& \leq C\|f\|_{L_{\rho, \theta}^{p, \kappa}\left(\mathbb{H}^{n}\right)} \sum_{k=2}^{\infty} \frac{1}{2^{k \delta}} \cdot\left(\frac{\left|B\left(u_{0}, 2^{k+1} r\right)\right|}{\left|B\left(u_{0}, r\right)\right|}\right)^{\beta / Q}\left(1+\frac{r}{\rho\left(u_{0}\right)}\right)^{N \cdot \frac{N_{0}}{N_{0}+1}}\left(1+\frac{2^{k+1} r}{\rho\left(u_{0}\right)}\right)^{-N+\theta} \\
& \leq C\|f\|_{L_{\rho, \theta}^{p, \kappa}\left(\mathbb{H}^{n}\right)} \sum_{k=2}^{\infty} \frac{1}{2^{k(\delta-\beta)}} \cdot\left(1+\frac{r}{\rho\left(u_{0}\right)}\right)^{N \cdot \frac{N_{0}}{N_{0}+1}}
\end{aligned}
$$

where $N>0$ is a sufficiently large number so that $N>\theta$. Also observe that $\beta<\delta \leq 1$, and hence the last series is convergent. Therefore,

$$
K_{2} \leq C\|f\|_{L_{\rho, \theta}^{p, \kappa}\left(\mathbb{H}^{n}\right)}\left(1+\frac{r}{\rho\left(u_{0}\right)}\right)^{N \cdot \frac{N_{0}}{N_{0}+1}} .
$$

Fix this $N$ and set $\vartheta=\max \left\{\theta, N \cdot \frac{N_{0}}{N_{0}+1}\right\}$. Finally, combining the above estimates for $K_{1}$ and $K_{2}$, the inequality (4.2) is proved and then the proof of Theorem 2.5 is finished.

In the end of this article, we discuss the corresponding estimates of the fractional integral operator $I_{\alpha}=\left(-\Delta_{\mathbb{H}^{n}}\right)^{-\alpha / 2}$ (under $0<\alpha<Q$ ). We denote by $K_{\alpha}^{*}(u, v)$ the kernel of $I_{\alpha}=\left(-\Delta_{\mathbb{H} n}\right)^{-\alpha / 2}$. In (1.10), we have already shown that

$$
\left|K_{\alpha}^{*}(u, v)\right| \leq C_{\alpha, n} \cdot \frac{1}{\left|v^{-1} u\right|^{Q-\alpha}} .
$$

Using the same methods and steps as we deal with (4.1) in Lemma 4.2, we can also show that, for some fixed $0<\delta \leq 1$ and $0<\alpha<Q$, there exists a positive constant $C_{\alpha, n}>0$ such 
that, for all $u, v$ and $w$ in $\mathbb{H}^{n}$,

$$
\left|K_{\alpha}^{*}(u, w)-K_{\alpha}^{*}(v, w)\right| \leq C_{\alpha, n} \cdot \frac{\left|v^{-1} u\right|^{\delta}}{\left|w^{-1} u\right|^{Q-\alpha+\delta}},
$$

whenever $\left|v^{-1} u\right| \leq\left|w^{-1} u\right| / 2$. Following along the lines of the proof of Theorems 2.3-2.5 and using the inequalities (4.5) and (4.6), we can obtain the following estimates of $I_{\alpha}$ with $\alpha \in(0, Q)$.

Theorem 4.3 Let $0<\alpha<Q, 1<p<Q / \alpha$ and $1 / q=1 / p-\alpha / Q$. If $0<\kappa<p / q$, then the fractional integral operator $I_{\alpha}$ is bounded from $L^{p, \kappa}\left(\mathbb{H}^{n}\right)$ into $L^{q,(\kappa q) / p}\left(\mathbb{H}^{n}\right)$.

Theorem 4.4 Let $0<\alpha<Q, p=1$ and $q=Q /(Q-\alpha)$. If $0<\kappa<1 / q$, then the fractional integral operator $I_{\alpha}$ is bounded from $L^{1, \kappa}\left(\mathbb{H}^{n}\right)$ into $W L^{q,(\kappa q)}\left(\mathbb{H}^{n}\right)$.

Here, we remark that Theorems 4.3 and 4.4 have been proved by Guliyev et al. [4].

Theorem 4.5 Let $0<\alpha<Q, 1<p<Q / \alpha$ and $1 / q=1 / p-\alpha / Q$. If $p / q \leq \kappa<1$, then the fractional integral operator $I_{\alpha}$ is bounded from $L^{p, \kappa}\left(\mathbb{H}^{n}\right)$ into $\mathcal{C}^{\beta}\left(\mathbb{H}^{n}\right)$ with $\beta / Q=\kappa / p-1 / q$ and $\beta<\delta \leq 1$, where $\delta$ is given as in (4.6).

As an immediate consequence we have the following corollary.

Corollary 4.6 Let $0<\alpha<Q, 1<p<Q / \alpha$ and $1 / q=1 / p-\alpha / Q$. If $\kappa=p / q$, then the fractional integral operator $I_{\alpha}$ is bounded from $L^{p, \kappa}\left(\mathbb{H}^{n}\right)$ into $\mathrm{BMO}\left(\mathbb{H}^{n}\right)$.

Upon taking $\alpha=1$ in Theorem 4.5, we get Morrey's lemma on the Heisenberg group.

Corollary 4.7 Let $\alpha=1,1<p<Q$ and $1 / q=1 / p-1 / Q$. If $p / q<\kappa<1$, then the fractional integral operator $I_{1}$ is bounded from $L^{p, \kappa}\left(\mathbb{H}^{n}\right)$ into $\mathcal{C}^{\beta}\left(\mathbb{H}^{n}\right)$ with $\beta / Q=\kappa / p-1 / q$ and $\beta<$ $\delta \leq 1$, where $\delta$ is given as in (4.6). From this, it follows that, for any given $f \in C_{c}^{1}\left(\mathbb{H}^{n}\right)$, i.e., $f$ is $C^{1}$-smooth with compact support in $\mathbb{H}^{n}$,

$$
\|f\|_{\mathcal{C}^{\beta}\left(\mathbb{H}^{n}\right)} \leq C\left\|\nabla_{\mathbb{H}^{n}} f\right\|_{L^{p, \kappa}\left(\mathbb{H}^{n}\right)},
$$

where $0<\kappa<1, p>(1-\kappa) Q, \beta=1-(1-\kappa) Q / p$ and the gradient $\nabla_{\mathbb{H}^{n}}$ is defined by

$$
\nabla_{\mathbb{H}^{n}}=\left(X_{1}, \ldots, X_{n}, Y_{1}, \ldots, Y_{n}\right) .
$$

Acknowledgements

The author would like to express his deep gratitude to the referee for his valuable comments and suggestions.

Funding

There is no funding to report for this submission.

Availability of data and materials

Not applicable.

Competing interests

The author declares that he has no competing interests.

Authors' contributions

The author read and approved the final manuscript. 


\section{Publisher's Note}

Springer Nature remains neutral with regard to jurisdictional claims in published maps and institutional affiliations.

Received: 7 June 2019 Accepted: 13 August 2019 Published online: 29 August 2019

\section{References}

1. Bongioanni, B., Harboure, E., Salinas, O.: Commutators of Riesz transforms related to Schrödinger operators. J. Fourier Anal. Appl. 17, 115-134 (2011)

2. Bongioanni, B., Harboure, E., Salinas, O.: Weighted inequalities for commutators of Schrödinger-Riesz transforms. J. Math. Anal. Appl. 392, 6-22 (2012)

3. Dziubański, J., Garrigós, G., Martínez, T., Torrea, J.L., Zienkiewicz, J.: BMO spaces related to Schrödinger operators with potentials satisfying a reverse Hölder inequality. Math. Z. 249, 329-356 (2005)

4. Guliyev, V.S., Eroglu, A., Mammadov, Y.Y.: Riesz potential in generalized Morrey spaces on the Heisenberg group. J. Math. Sci. (N.Y.) 189, 365-382 (2013)

5. Jerison, D., Sanchez-Calle, A.: Estimates for the heat kernel for a sum of squares of vector fields. Indiana Univ. Math. J. 35, 835-854 (1986)

6. Jiang, Y.S.: Some properties of the Riesz potential associated to the Schrödinger operator on the Heisenberg groups. Acta Math. Sin. (Chin. Ser.) 53, 785-794 (2010)

7. Jiang, Y.S.: Endpoint estimates for fractional integral associated to Schrödinger operators on the Heisenberg groups. Acta Math. Sci. Ser. B 31, 993-1000 (2011)

8. Lin, C.C., Liu, H.P.: $B M O_{L}\left(\mathbb{H}^{n}\right)$ spaces and Carleson measures for Schrödinger operators. Adv. Math. 228, 1631-1688 (2011)

9. Lu, G.Z:: A Fefferman-Phong type inequality for degenerate vector fields and applications. Panam. Math. J. 6, 37-57 (1996)

10. Shen, Z.W.: $L^{p}$ estimates for Schrödinger operators with certain potentials. Ann. Inst. Fourier (Grenoble) $45,513-546$ (1995)

11. Stein, E.M.: Singular Integrals and Differentiability Properties of Functions. Princeton University Press, Princeton (1970)

12. Stein, E.M.: Harmonic Analysis: Real-Variable Methods, Orthogonality, and Oscillatory Integrals. Princeton University Press, Princeton (1993)

13. Thangavelu, S.: Harmonic Analysis on the Heisenberg Group. Progress in Mathematics, vol. 159. Birkhäuser, Basel (1998)

14. Xiao, J.S., He, J.X.: Riesz potential on the Heisenberg group. J. Inequal. Appl. 2011, Article ID 498638 (2011)

\section{Submit your manuscript to a SpringerOpen ${ }^{\circ}$ journal and benefit from:}

- Convenient online submission

- Rigorous peer review

- Open access: articles freely available online

- High visibility within the field

- Retaining the copyright to your article

Submit your next manuscript at $\boldsymbol{~ s p r i n g e r o p e n . c o m ~}$ 